\title{
VEGF, FLT3 ligand, PIGF and HGF can substitute for $M-C S F$ to induce human osteoclast formation: implications for giant cell tumour pathobiology
}

\author{
Richard M Taylor, Takeshi G Kashima, Helen J Knowles and Nicholas A Athanasou
}

\begin{abstract}
Giant cell tumour of bone (GCTB) is a primary bone tumour that contains numerous very large, hyper-nucleated osteoclastic giant cells. Osteoclasts form from CD14 + monocytes and macrophages in the presence of receptor activator of nuclear factor kappa B ligand (RANKL) and macrophage-colony stimulating factor (M-CSF). GCTB contains numerous growth factors, some of which have been reported to influence osteoclastogenesis and resorption. We investigated whether these growth factors are capable of substituting for M-CSF to support osteoclast formation from cultured human monocytes and whether they influence osteoclast cytomorphology and resorption. Vascular endothelial growth factor-A (VEGF-A), VEGF-D, FLT3 ligand (FL), placental growth factor (PIGF) and hepatocyte growth factor (HGF) supported RANKL-induced osteoclastogenesis in the absence of M-CSF, resulting in the formation of numerous TRAP + multinucleated cells capable of lacunar resorption. Monocytes cultured in the presence of M-CSF, HGF, VEGF-A and RANKL together resulted in the formation of very large, hyper-nucleated (GCTB-like) osteoclasts that were hyper-resorptive. M-CSF and M-CSF substitute growth factors were identified immunohistochemically in GCTB tissue sections and these factors stimulated the resorption of osteoclasts derived from a subset of GCTBs. Our findings indicate that there are growth factors that are capable of substituting for M-CSF to induce human osteoclast formation and that these factors are present in GCTB where they influence osteoclast cytomorphology and have a role in osteoclast formation and resorption activity.

Laboratory Investigation (2012) 92 1398-1406; doi:10.1038/labinvest.2012.108; published online 20 August 2012
\end{abstract}

KEYWORDS: FLT3 ligand; HGF; M-CSF; osteoclast; PIGF; VEGF

The osteoclast is a multinucleated cell, which is formed from circulating mononuclear phagocyte precursors derived from the bone marrow. ${ }^{1,2}$ It exhibits a number of specialised cytochemical and functional features, including the ability to carry out lacunar bone resorption. Increased osteoclast formation and activity is seen in a number of osteolytic bone and joint conditions, including notably giant cell tumour of bone (GCTB), a primary bone tumour, which contains numerous, very large, often hyper-nucleated osteoclastic giant cells that effect a considerable amount of bone resorption. ${ }^{3,4}$

Osteoclast differentiation from monocyte or macrophage precursors requires the presence of receptor activator of nuclear factor kappa B ligand (RANKL) and macrophage-colony stimulating factor (M-CSF). ${ }^{5}$ The importance of M-CSF in osteoclast formation is evidenced by the fact that in op/op osteopetrotic mice, which have a mutation in the M-CSF gene, very few osteoclasts are found in bone and there is markedly decreased bone resorption. ${ }^{6,7} \mathrm{M}$-CSF promotes several aspects of osteoclastogenesis including the proliferation and fusion of osteoclast precursors as well as osteoclasts and the expression of the RANKL receptor by these cells. ${ }^{8-10}$ The effect of M-CSF on mature osteoclast resorption activity is controversial with both a decrease and increase in lacunar resorption being reported. ${ }^{11-13}$

Although bone resorption is greatly decreased in op/op mice, a few osteoclasts can still be found in skeletal tissues; op/op mice can also undergo a spontaneous age-dependent recovery of osteoclastogenesis. ${ }^{14,15}$ These observations suggest that one or more growth factors may be capable of substituting for M-CSF to induce osteoclast formation. This was confirmed in several studies where hepatocyte growth factor (HGF), vascular endothelial growth factor-A

Nuffield Department of Orthopaedics, Rheumatology and Musculoskeletal Science, University of Oxford, Nuffield Orthopaedic Centre, Oxford, UK Correspondence: Professor NA Athanasou, Nuffield Department of Orthopaedics, Rheumatology and Musculoskeletal Science, University of Oxford, Nuffield Orthopaedic Centre, Oxford, OX3 7LD, UK.

E-mail: nick.athanasou@ndorms.ox.ac.uk

Received 19 January 2012; revised 14 May 2012; accepted 23 May 2012 
(VEGF-A), placental growth factor (PlGF) and FLT3 ligand (FL) were shown to be capable of supporting osteoclastogenesis in the absence of M-CSF. ${ }^{16-19}$ These growth factors are abundant and widely distributed in human tissues and are thought to have a role in several neoplastic and inflammatory conditions, which affect bone and joint, including GCTB. ${ }^{20}$

The effect of potential M-CSF substitute growth factors on human osteoclast formation and resorption is not well defined. There is contradictory evidence as to whether VEGF and FL independently support osteoclastogenesis. ${ }^{17,20-22}$ In this study we have analysed the effect of M-CSF and M-CSF substitute growth factors, both alone and in combination, on human monocyte-osteoclast differentiation. In addition, we have examined whether these growth factors are expressed in GCTB and whether they influence osteoclast cytomorphology and resorption activity.

\section{MATERIALS AND METHODS \\ Reagents}

For all cultures alpha minimal essential medium ( $\alpha$-MEM) (Lonza, Wokingham, UK) was supplemented with $10 \%$ heatinactivated FBS, $50 \mathrm{IU} / \mathrm{ml}$ Penicillin, $50 \mu \mathrm{g} / \mathrm{ml}$ Streptomycin (Invitrogen, Paisley, UK) and $2 \mathrm{mM}$ L-Glutamine (Lonza) (MEM/FCS). Human-M-CSF and anti-M-M-CSF antibody were purchased from R\&D Systems (Abingdon, UK), RANKL and osteoprotegrin (OPG) from Peprotech (London, UK) and calcitonin from Sigma (Poole, UK). Imatinib and Sunitinib were purchased from Cayman Chemical (Ann Arbour, MI, USA) and BioVision (Milpitas, CA, USA), respectively. All cultures were maintained at $37^{\circ} \mathrm{C}$ in $5 \% \mathrm{CO}_{2}$.

\section{Isolation and Culture of Human Monocytes Derived from Peripheral Blood}

Human monocytes were obtained by density gradient centrifugation of $50 \mathrm{ml}$ of buffy coat cell preparation provided by the National Blood Transfusion Service (Bristol, UK). The buffy coat preparation was mixed with an equal volume of MEM and purified over Histopaque (Sigma-Aldrich, Dorset, UK). After centrifugation at 2250 r.p.m. for $25 \mathrm{~min}$, the cell layer above the Histopaque was collected, suspended in MEM, and centrifuged at 1800 r.p.m. for $10 \mathrm{~min}$. The cell pellet was resuspended in MEM and centrifuged again. Five milliliter of MEM/FBS was then added to the cell pellet and the number of cells counted in a haematocytometer following lysis of red blood cells with $5 \%(\mathrm{v} / \mathrm{v})$ acetic acid. $5 \times 10^{5}$ cells per well were plated immediately onto dentine slices and glass coverslips in 96-well tissue culture plates with $100 \mu \mathrm{l} /$ well of MEM/FBS. After $3 \mathrm{~h}$ incubation, the dentine slices and glass coverslips were washed in MEM/FBS to remove any non-adherent cells, and transferred into 24-well tissue culture plates containing $1 \mathrm{ml}$ of MEM/FBS. CD14+ monocytes were cultured on coverslips and dentine slices for 14 and 21 days, respectively, in $1 \mathrm{ml}$ of $\alpha-M E M \pm$ RANKL $(50 \mathrm{ng} / \mathrm{ml})$ in the presence and absence of the following growth factors, either alone or in combination and with/ without added anti-M-CSF antibody ( $100 \mathrm{ng} / \mathrm{ml})$; M-CSF ( $25 \mathrm{ng} / \mathrm{ml})$; VEGF-A $(25 \mathrm{ng} / \mathrm{ml})$; VEGF-C $(25 \mathrm{ng} / \mathrm{ml})$; VEGF-D $(25 \mathrm{ng} / \mathrm{ml}) ;$ PlGF $(25 \mathrm{ng} / \mathrm{ml}) ;$ FL ( $25 \mathrm{ng} / \mathrm{ml}) ;$ HGF $(25 \mathrm{ng} / \mathrm{ml})$.

\section{Cytochemical and Immunophenotypic Assessment of Osteoclast Formation}

Following incubation for 14 days, monocyte cultures on coverslips were fixed and stained for TRAP, and immunocytochemically for CD14 and CD51 as previously described. ${ }^{2,4}$ Multinucleated cells expressing TRAP and containing more than three nuclei were considered osteoclasts. Cell cultures and coverslips were fixed in acetone. Endogenous peroxidase activity was blocked with $3 \%$ hydrogen peroxide and a protein serum block (Dako, UK). This was followed by incubation with the monoclonal antibodies 23C6 (Serotec, Oxford, UK) and NCL-CD14 (Leica, UK) directed against CD51 and CD14 respectively.

\section{Functional Characterisation of Human Osteoclasts}

To determine the presence of $\mathrm{F}$-actin rings, which provide evidence of osteoclast attachment to a mineralised substrate, 21-day monocyte cultures on dentine slices were fixed in $4 \%$ formalin, permeabilized with $0.5 \%$ Triton X-100 and stained in the dark with TRITC-conjugated phalloidin $(0.5 \mu \mathrm{g} / \mathrm{ml})$ and DAPI $(20 \mathrm{ng} / \mathrm{ml})$ (Sigma). Cells were visualised using a fluorescence microscope.

Evidence of lacunar resorption was determined in 21-day monocyte cultures on dentine slices. Adherent cells were removed from dentine slices following treatment with $1 \mathrm{~N}$ $\mathrm{NH}_{4} \mathrm{OH}$ and sonication. Lacunar resorption pits were visualised by light microscopy after staining with $0.5 \%(\mathrm{w} / \mathrm{v})$ toluidine blue in $0.5 \%$ aqueous boric acid $(\mathrm{w} / \mathrm{v})(\mathrm{pH} 7.2-$ 7.4). The mean area resorbed was determined using Adobe Photoshop and Image J (National Institutes of Health, USA) software as previously described. ${ }^{4}$

\section{Effect of M-CSF and M-CSF Substitutes on Mature Osteoclast Resorption}

To investigate the effect of M-CSF and M-CSF substitutes on mature osteoclast resorption, osteoclasts were isolated directly from 11 GCTBs by curettage, as previously described ${ }^{3,4}$ and cultured on dentine slices for $48 \mathrm{~h}$ in the presence of M-CSF $(25 \mathrm{ng} / \mathrm{ml})$, HGF $(25 \mathrm{ng} / \mathrm{ml})$, VEGF $(25 \mathrm{ng} / \mathrm{ml})$, FL $(25 \mathrm{ng} / \mathrm{ml}), \operatorname{PlGF}(25 \mathrm{ng} / \mathrm{ml})$ and RANKL $(50 \mathrm{ng} / \mathrm{ml})$, either alone or in combination. In addition, osteoclasts were isolated directly from two GCTBs and cultured on dentine slices for $48 \mathrm{~h}$ in the presence of growth factor receptor inhibitors including those for M-CSF and VEGF (Imatinib, $5 \mathrm{nM}$ and Sunitinib, $1 \mathrm{nM}$ ).

Following incubation, the dentine slices were treated with $1 \mathrm{~N} \mathrm{NH}_{4} \mathrm{OH}$, stained with toluidine blue and the lacunar resorption surface area quantitifed as detailed above. Lacunar resorption values were expressed as a percentage of resorption relative to the plain control (ie, osteoclasts cultured 
in MEM/FCS with no added factors.). To ensure that the lacunar resorption data reflected osteoclast resorption activity and not a change in osteoclast number, the number of giant cells was counted after $48 \mathrm{~h}$ of incubation. No difference in osteoclast number was noted between experimental conditions and the plain control.

\section{Immunophenotypic Expression of M-CSF and M-CSF Substitutes in GCTB}

Five microns sections were prepared from formalin-fixed, paraffin-embedded specimens of seven cases of GCTB received at the Nuffield Orthopaedic Centre, Oxford and from a tissue microarray (provided by Professor Burger, Westfalische Wilhmsuniversitai, Munster, Germany) containing tissue from 107 GCTB samples. These included conventional, locally aggressive tumours, two of which had resulted in 'metastatic' lung nodules. Malignant GCTBs were excluded from this study. Expression of M-CSF, HGF, VEGF, FL and PlGF was determined by immunohistochemistry using an indirect immunoperoxidase technique. M-CSF was detected using a rabbit monoclonal anti-M CSF antibody (ab528564, Abcam, Cambridge, UK) at 1:50 dilution for 1 h; HGF was detected using a goat polyclonal antibody (AF-294-NA, R\&D Systems), at 1:100 dilution overnight; VEGF-A was detected using a mouse monoclonal antibody (MAB293, R\&D Systems), at a 1:100 dilution overnight. FL was detected using a rabbit monoclonal antibody (ab-52648, Abcam), at a dilution of 1:100 for $1 \mathrm{~h}$. PlGF was detected using a rabbit polyclonal antibody (ab-9542, Abcam), at a dilution of 1:500 for $1 \mathrm{~h}$. RANKL was detected using a goat polyclonal antibody (sc-7627, Santa Cruz Biotechnology, USA), at $25 \mu \mathrm{g} / \mathrm{ml}$ for $1 \mathrm{~h}$.

\section{Messenger RNA (mRNA) Expression of M-CSF and M-CSF Substitutes}

To confirm mRNA expression of M-CSF and M-CSF substitutes in GCTB tissue, real-Time PCR (RT-PCR) was carried out to identify mRNA transcripts for M-CSF, VEGF-A, VEGF-D, PlGF, FL and HGF. Total RNA was extracted from tissue samples using RNAeasy (Qiagen) and treated with DNAase I (Invitrogen). Single-stranded cDNA was synthesised using the SuperScript VILO cDNA Synthesis Kit (Invitrogen). RT-PCR was performed with the Rotor-Gene 3000 (Corbett Research) and the Express SYBR GreenER Kit (qPCR Supermix Universal) (Invitrogen). The PCR cycling conditions were as follows: $50^{\circ} \mathrm{C} 2 \mathrm{~min}, 95^{\circ} \mathrm{C} 2 \mathrm{~min}$, $95^{\circ} \mathrm{C} 15 \mathrm{~s}, 60^{\circ} \mathrm{C} 1 \mathrm{~min}$ for 40 cycles. QuantiTect real-time primer sets were designed by Qiagen. The catalogue numbers were as follows; M-CSF (QT00035224), VEGF-A (QT01682072), VEGF-D (QT00003997), FL (QT00000245), P1GF (QT00030688), HGF (QT01758988) and G3PDH (glyceraldehyde-3-phosphate dehydrogenase, QT01192646). G3PDH mRNA expression was used as a control. Positive control for cytokine mRNA expression was reactive lymph node tissue.

\section{Statistical Analysis}

Statistical significance was determined using Student's $t$-test for comparing paired samples or one-way ANOVA followed by Dunnet post hoc test for comparing all sample groups against one control group. $P$ values $<0.05$ were considered significant. All figures are derived from at least three independent experiments and are plotted as mean \pm s.d. In order to minimise the effect of batch-to-batch variation of monocytes, TRAP and lacunar resorption data were normalised to the number of TRAP + cells and lacunar resorption, respectively, noted in the positive control (ie, monocytes incubated with M-CSF $(25 \mathrm{ng} / \mathrm{ml})$ and RANKL $(50 \mathrm{ng} / \mathrm{ml}))$. Experiments were performed in triplicate unless otherwise stated.

\section{Results M-CSF-Independent Osteoclast Formation}

RANKL-treated cultures of monocytes incubated in the absence of M-CSF but in the presence of VEGF-A, VEGF-D, PIGF, FL or HGF alone resulted in the formation of TRAP + I $\mathrm{CD} 51+$ multinucleated cells; these cultures also, unlike monocytes, formed F-actin rings and were capable of producing lacunar resorption pits when cultured on dentine slices (Figure 1). Osteoclasts formed in the presence of VEGF-A, PIGF, FL and HGF were smaller and contained fewer nuclei than those formed in M-CSF-treated cultures. All osteoclasts generated in the presence of M-CSF and M-CSF substitutes formed large, single or compound lacunar resorption pits. Morphologically, the lacunar resorption pits were identical to pits observed in the positive control cultures. No evidence of osteoclast formation was noted when monocytes were cultured in the presence of VEGF-C and RANKL, RANKL, M-CSF or any of the M-CSF substitutes alone.

A concentration (5-200 ng/ml) curve for each of the above M-CSF substitute growth factors was analysed to determine the concentration, which stimulated maximal osteoclast formation and resorption. The optimal concentration for the M-CSF substitutes was as follows: VEGF-A, VEGF-D and HGF $(10 \mathrm{ng} / \mathrm{ml})$, and FL and PlGF $(50 \mathrm{ng} / \mathrm{ml})$. The amount of resorption measured in VEGF-A, VEGF-D, PIGF, FL and HGF cultures was 4.5, 5.4, 7.5, 4.9 and $12.5 \%$ relative to the positive control (M-CSF/RANKL-treated cultures); the addition of an M-CSF neutralising antibody to the monocyte cultures did not inhibit the formation TRAP +/CD51+ multinucleated cells or lacunar resorption pit formation (Figure 2).

To investigate if combinations of M-CSF substitute growth factors influenced osteoclast formation, monocytes were cultured in the presence of RANKL and all possible combinations of M-CSF, VEGF-A, PlGF, FL and HGF (added at optimal concentration). It was found that in the presence of M-CSF, VEGF-A, HGF and RANKL (MVHR), numerous, very large, hyper-nucleated TRAP + giant cells formed on coverslips; these multinucleated cells were hyper-resorptive, 

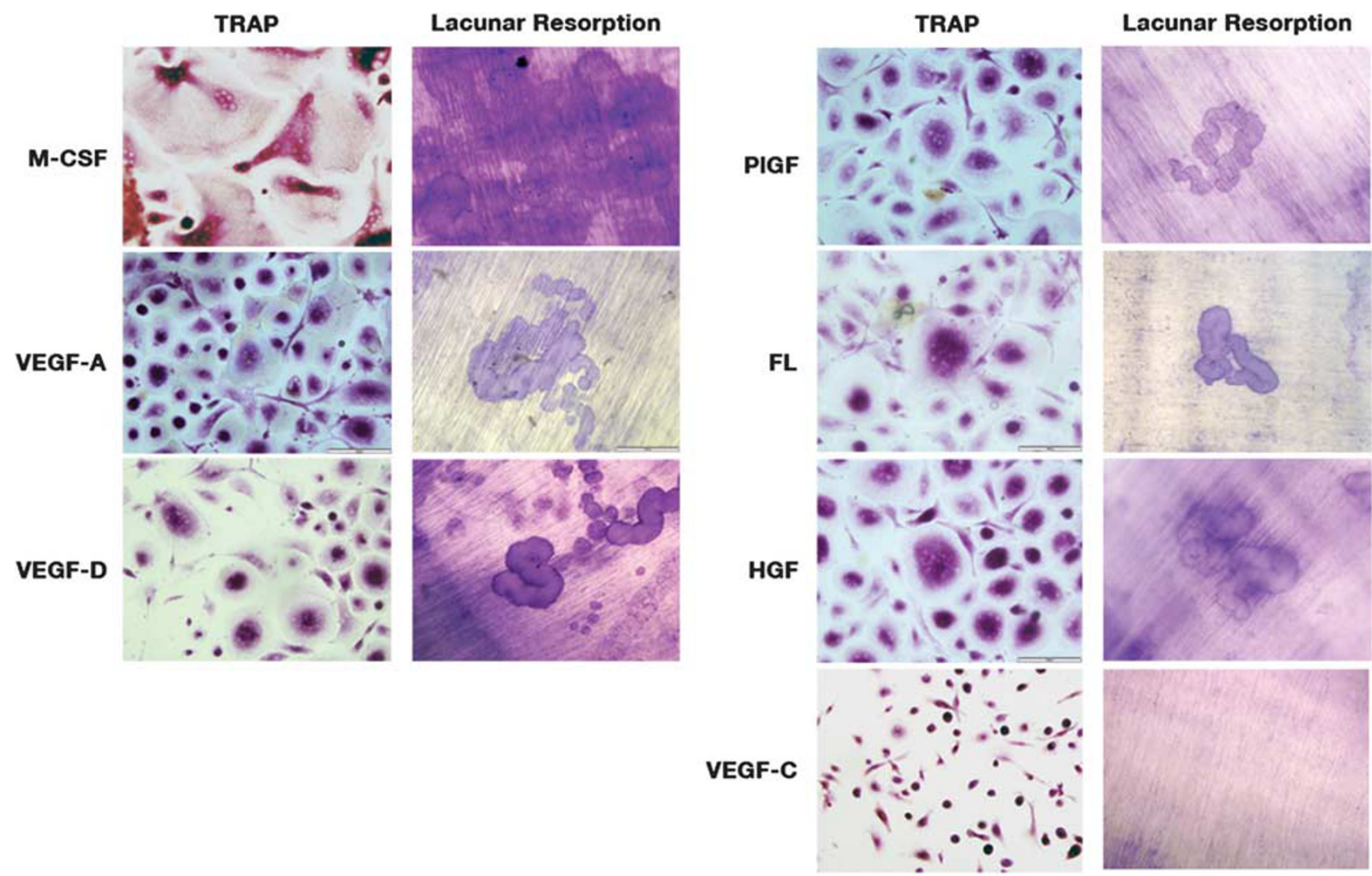

Figure 1 Formation of TRAP + multinucleated cells and lacunar resorption pits in monocyte cultures incubated in the presence of RANKL and M-CSF, VEGF-A, VEGF-D, PIGF, FL and HGF but not VEGF-C.

effecting $72 \%$ more resorption than M-CSF and RANKLtreated cultures (Figure 3). Osteoclasts formed in MVHRtreated monocyte cultures were considerably larger and contained more nuclei than osteoclasts formed in cultures containing all other possible combinations of growth factors (including the positive control). The addition of PlGF and FL to MVHR cultures did not affect the morphology or number of TRAP + multinucleated cells observed in 14-day monocyte cultures or the extent of lacunar resorption in 21-day cultures.

\section{Immunophenotypic and mRNA Expression of Osteoclastogenic Growth Factors in GCTB}

Expression of M-CSF, HGF, VEGF-A, FL and PlGF was noted in GCTB tissue sections by immunohistochemistry (Figure 4). Expression of these growth factors was noted in mononuclear and giant cell populations in most tumours with M-CSF, HGF and RANKL being identified mainly in mononuclear cells and FL, PlGF and VEGF commonly observed in giant cells. No correlation was seen in expression of the above growth factors with regard to the morphological characteristics of the GCTB (eg, number of giant cells, mitotic activity of mononuclear cells, vascular invasion) on tumour behaviour (eg, tumour growth/size, extent of locally aggressive behaviours or presence of lung nodules). RT-PCR

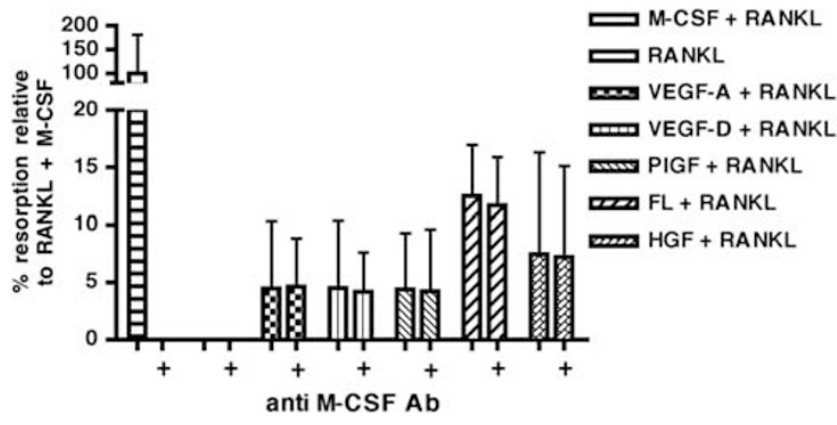

Figure 2 Mean percentage area lacunar resorption relative to the positive control (M-CSF and RANKL) of human monocytes cultured with the M-CSF substitutes with statistical significance $(P<0.01$, Dunnet post hoc test following one-way ANOVA). The addition of an anti-M-CSF neutralising antibody abolished lacunar resorption in control cultures, but not cultures containing M-CSF substitutes. Mean + s.d.

studies showed variable expression of M-CSF and M-CSF substitutes in two cases of GCTB (Figure 5).

\section{Effect of M-CSF and M-CSF Substitute Growth Factors on Lacunar Resorption by GCTB-Derived Osteoclasts}

The addition of M-CSF or the M-CSF substitutes alone to GCTB-derived osteoclasts cultured on dentine slices had no effect on total lacunar resorption when all GCTB samples were analysed together. However, in a subset of GCTBs, the 

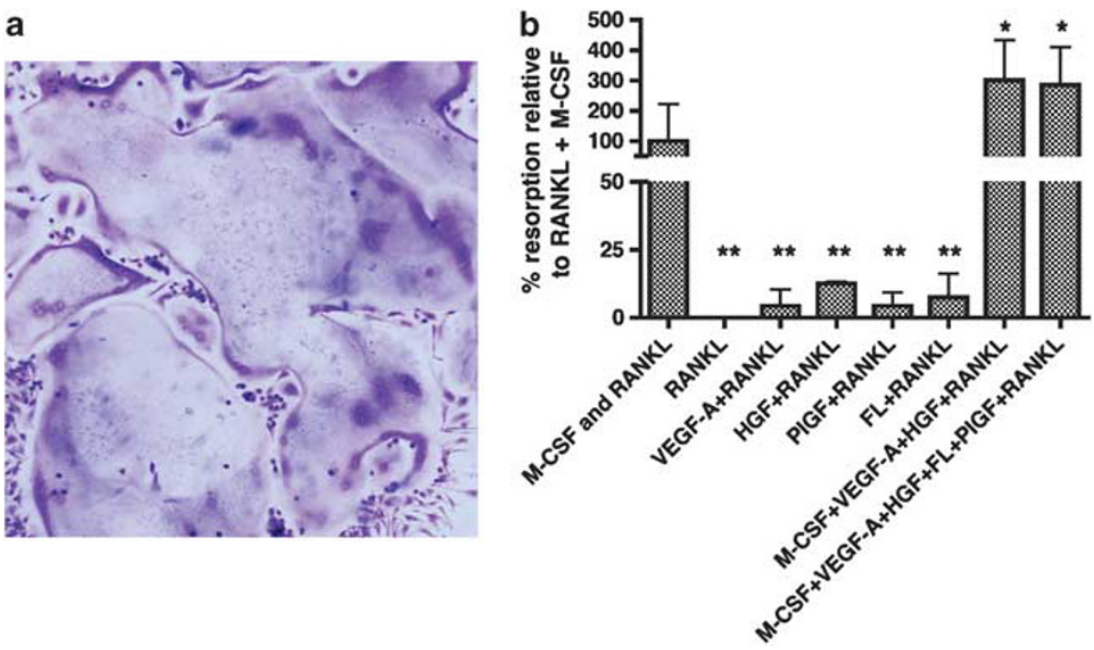

Figure 3 (a) TRAP + multinucleated cells formed in cultures of monocytes incubated with M-CSF, HGF, VEGF and RANKL (MHVR) showing large multinucleated cells. (b) Mean percentage area lacunar resorption in monocyte cultures incubated with M-CSF, VEGF, HGF, RANKL ( \pm PIGF and FL). Lacunar resorption in MHVR-treated cultures was greater than with M-CSF and RANKL alone. The addition of FL and PIGF to MHVR-treated cultures did not increase lacunar resorption. Mean + s.d. ${ }^{*} P<0.05(n=7)$.
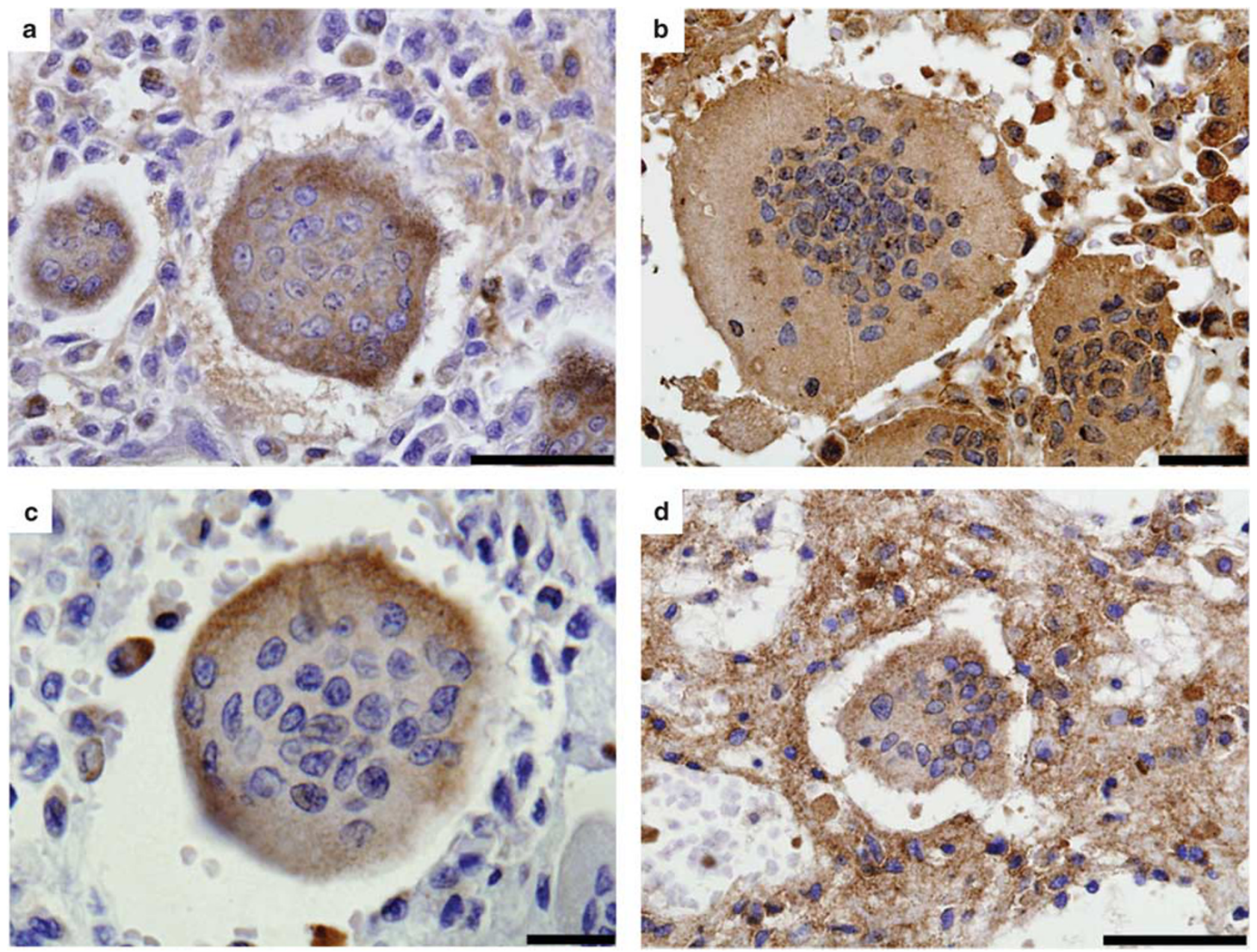

Figure 4 Immunohistochemical detection of M-CSF and M-CSF substitutes in GCTB showing expression of M-CSF (a), VEGF-A (b), PIGF (c) and HGF in multinucleated and mononuclear cell populations. Scale bar $=20 \mu \mathrm{m}$. 


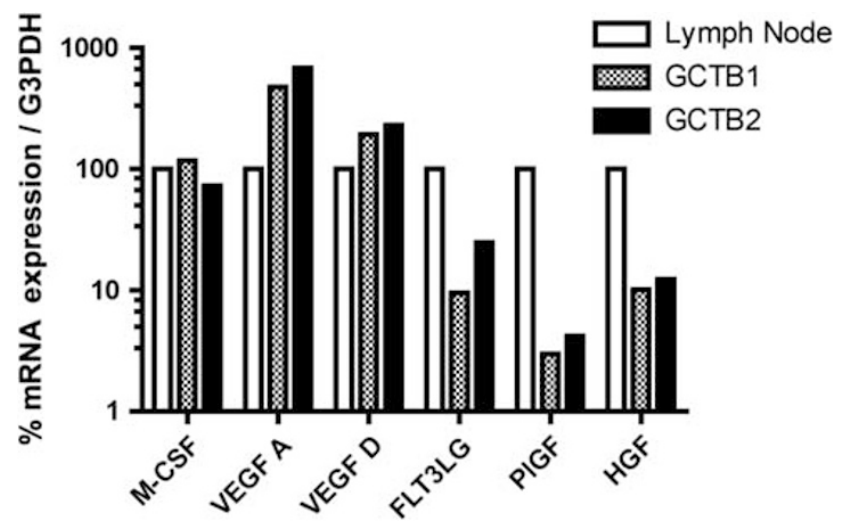

Figure 5 Expression of M-CSF and M-CSF substitute mRNA transcripts in two cases of GCTB and control reactive lymph node tissue. Data standardised to G3PDH mRNA expression level.

addition of M-CSF or the M-CSF substitute alone enhanced resorption relative to the untreated control (104.5-264.2\%, data not shown). In the other eight tumours, the addition of M-CSF and M-CSF substitutes did not increase lacunar resorption. The addition of RANKL markedly increased lacunar resorption by osteoclastic giant cells derived from all tumours. The additions of inhibitors to Imatinib $(5 \mathrm{nM})$ and Sunitinib ( $1 \mathrm{nM})$ suppressed lacunar resorption and show no cytotoxic effect on osteoclasts (Figure 6).

\section{DISCUSSION}

In this study we have shown that the growth factors VEGF-A, VEGF-D, PlGF, FL and HGF are capable of substituting for M-CSF to support RANKL-induced human monocyte-osteoclast differentiation. Multinucleated cells expressing the cytochemical and functional markers of osteoclasts were formed when these growth factors were added to monocyte cultures, even in the presence of an anti-M-CSF neutralising antibody. Osteoclast resorption was less than that seen in M-CSF and RANKL-treated cultures but a marked increase in osteolysis was noted when VEGF and HGF were added to M-CSF/RANKL-treated monocyte cultures with the formation of giant, hyper-multinucleated and hyper-resorptive osteoclasts, similar to those seen in GCTB.

Multinucleated cells which formed in $\mathrm{CD} 14+$ monocyte cultures to which VEGF-A, VEGF-D, PlGF, FL or HGF were added expressed characteristic markers of mature osteoclasts including TRAP, CD51, F-actin rings and the ability to carry out lacunar resorption. These findings suggest that these growth factors may have a role in compensating for the absence of M-CSF in op/op mice, which have a mutation in the coding region of the M-CSF gene that results in an osteopetrotic phenotype. ${ }^{6,7}$ It is known that a few osteoclasts can be identified in bones of these osteopetrotic mice and that this defect, can partially correct itself with age. ${ }^{14,15}$ The above growth factors may thus provide a salvage pathway for osteoclast formation that could explain this observation. The cytokine Interleukin 34, which has also recently been shown to bind to the M-CSF receptor and to have a role in osteoclastogenesis, is also expressed in GCTB. ${ }^{23,24}$ The cytokine interleukin 34 , which has also recently been shown to react with the M-CSF receptor and to have a role in osteoclastogenesis, is also expressed in GCTB. ${ }^{23,24}$

Conflicting evidence exists as to whether VEGF-A can support osteoclast formation. ${ }^{17-19,21,22}$ Our findings are in agreement with studies showing that VEGF-A can correct the defect in op/op mice and support a role for VEGF-A in osteoclast formation. Although VEGF-A has previously been reported to support human osteoclast formation in vitro by Aldridge et $a l^{21}$ this was not conclusively shown as osteoclastogenesis was not determined in the presence of an anti-M-CSF antibody and resorption was noted in negative control cultures. This study also noted that VEGF-A- and M-CSF-induced osteoclastogenesis effected a similar level of resorption. We noted that osteoclasts formed in VEGF-Atreated cultures were capable of much less resorption $(7.5 \%$ relative to M-CSF-treated cultures) and did not observe a difference in the number of TRAP + multinucleated cells. Osteoclast formation and lacunar resorption in our M-CSFtreated cultures was considerably more $(65-80 \%$ compared with $4-8 \%$ ) than in the study of Aldridge et al. ${ }^{21} \mathrm{FL}$ has previously been shown in vitro to support osteoclast formation independently of M-CSF in mice (including op/ op mice), but not in man. ${ }^{17,21}$ Our findings indicate that FL can support human osteoclast formation; less resorption and fewer osteoclasts were observed compared with M-CSFtreated cultures. Our findings also confirm previous studies, which showed that HGF can support human osteoclast formation. ${ }^{16}$

Aldridge $e t a l^{21}$ also reported that VEGF-D and PlGF are capable of substituting for M-CSF in osteoclast formation. They found that, relative to VEGF-A, significantly fewer resorption pits were formed in VEGF-D- and PlGF-treated cultures, and that in VEGF-D treated cultures this was associated with fewer TRAP + cells. In the present study we did not observe a significant difference in the number of TRAP + cells or lacunar resorption in VEGF-D- or PlGFtreated cultures compared with VEGF-A-treated cultures. PIGF has been shown to have a role in bone remodelling and osteoclast differentiation. ${ }^{25}$ The addition of an anti-PIGF antibody has been shown to reduce the osteolysis in metastatic tumours. ${ }^{26}$ PIGF is a homologue of VEGF-C; both are RANKL targets and mediate RANKL activity. ${ }^{27} \mathrm{We}$ found, however, that VEGF-C was not able to support human osteoclast formation independent of M-CSF. RANKL enhances the expression of VEGF-C mRNA and overexpression of VEGF-C increases resorption activity four-fold. ${ }^{27}$ VEGF-C binds to VEGFR2 and VEGFR3, and osteoclasts have been shown to express VEGFR3; it is possible that VEGF-C mediates its effects largely through VEGFR2 rather than VEGFR3.

In general, VEGF-A, VEGF-D, PIGF, FL and HGF exhibited a similar capacity to act as M-CSF substitutes in osteoclast 

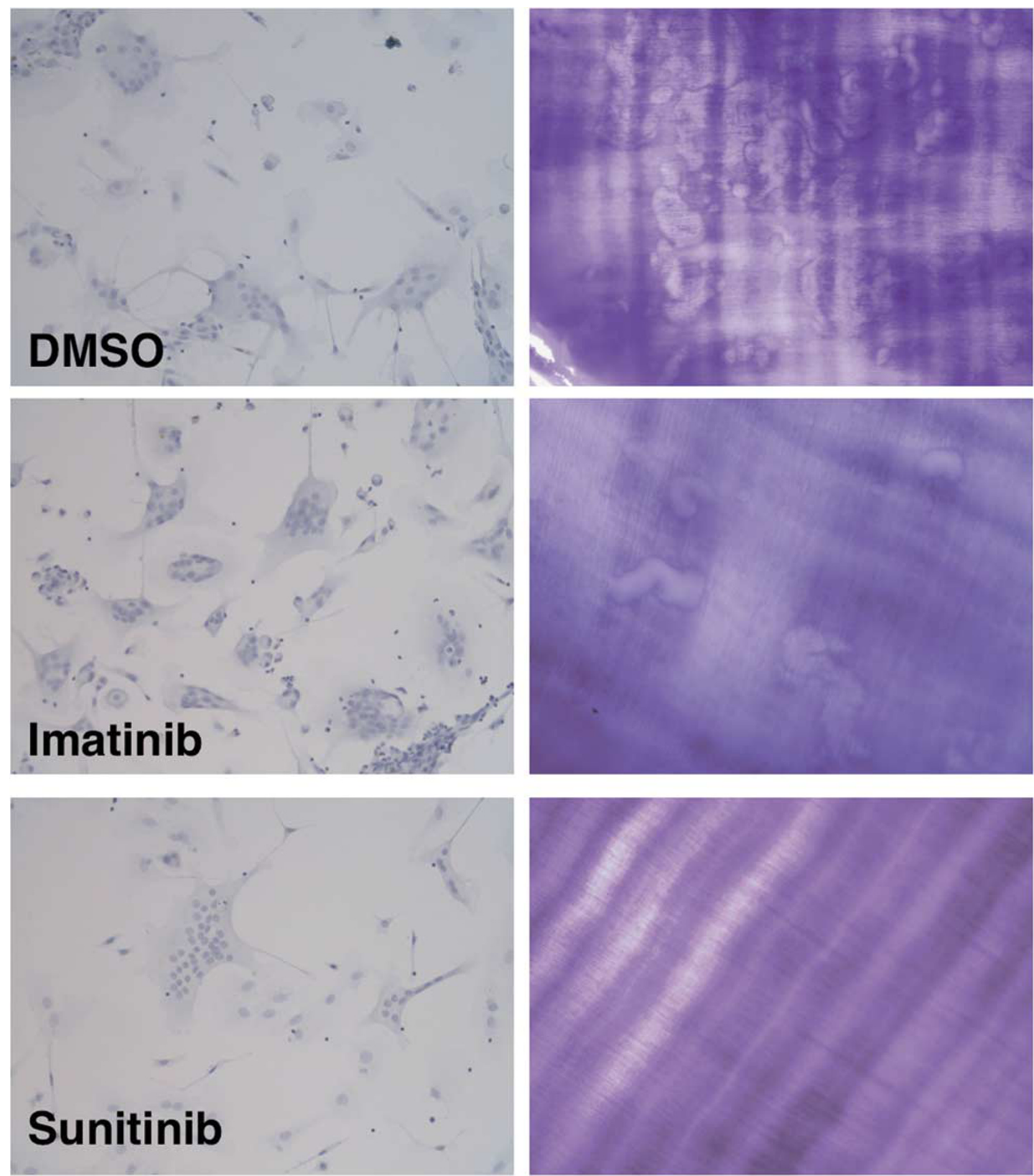

Figure 6 Inhibition of lacunar resorption pit formation in cultures of GCTB-derived osteoclasts treated with Imatinib (5 nm) and Sunitinib (1 nM).

formation: these growth factors produced similar numbers of TRAP + multinucleated cells that effected a similar amount of lacunar resorption. When VEGF-A, HGF and M-CSF were added to monocyte cultures, however, this resulted in the formation of giant, hyper-nucleated osteoclasts, which showed markedly increased lacunar resorption (1.7-fold more than control M-CSF/RANKL-treated cultures). The very large hyper-nucleated osteoclasts formed in the presence of MVHR were similar in cytomorphology to the osteoclastic giant cells of GCTB, a tumour which is markedly osteolytic. Immunohistochemical findings confirmed that M-CSF, HGF and VEGF (as well as FL and PlGF) are all present in GCTB. 
The presence of these osteoclastogenic growth factors may in part explain the giant cell-rich nature and aggressive osteolysis of GCTB. Both the multinucleated and mononuclear cell population in GCTB expressed M-CSF and M-CSF substitutes. The expression of osteoclastogenic factors by giant cells may represent a positive feedback autocrine mechanism that promotes osteoclast recruitment and formation in GCTB. ${ }^{28}$ Expression of osteoclastogenic factors by GCTB mononuclear cells is in keeping with the hypothesis that these cells express growth factors promoting osteoclast formation. ${ }^{20,29}$ VEGF-A and RANKL expression by GCTB giant cells and M-CSF expression in enriched cultures of GCTB giant cells has been reported in previous studies. ${ }^{30}$

Previous work has shown that M-CSF can inhibit or stimulate osteoclast resorption activity depending on the resorption model and species of osteoclast employed. ${ }^{11-13,31}$ The effect of both M-CSF and M-CSF substitutes on GCTBderived osteoclasts has not been previously investigated. M-CSF is known to stimulate osteoclast migration and it has been proposed that this is associated with the disassembly of the resorption machinery. ${ }^{32}$ We found that M-CSF alone did not inhibit the resorption activity of osteoclasts derived from most GCTBs. In fact, a stimulatory effect on resorption was seen in a minority of GCTBs, which clinically and morphologically were not obviously different from other (unstimulated) GCTBs. M-CSF substitutes also had a stimulatory effect on resorption in this group of tumours. Little work has been done on the effect of the M-CSF substitute growth factors on osteoclast resorption. VEGF has been shown to enhance resorption in cultures of rabbit osteoclasts $^{33}$ and HGF in rat osteoclast-osteoblast cocultures, whereas FL did not effect murine osteoclast resorption activity. ${ }^{34}$ Our preliminary results show an inhibitory effect of Imatinib and Sunitinib on osteoclast resorption in short-term cultures of two giant cell tumours.

The complex growth factor environment found in GCTB (and other giant cell-rich lesions of bone and joint) is likely to have a role in determining the pathological features and the extent of resorption, which characterise this tumour. Our findings indicate that VEGF-A, VEGF-D, PlGF, FL and HGF are capable of acting as M-CSF substitutes in osteoclastogenesis. These growth factors are expressed in GCTB and in specific growth factor combinations, possibly in concert with IL-34, ${ }^{23}$ may result in the formation of GCTB-like hypernucleated osteoclasts capable of lacunar resorption. M-CSF and M-CSF substitute growth factors also stimulated osteoclast resorption in a subset of GCTBs. In addition to other features of GCTB, such as increased vascularity and tumour progression ${ }^{20}$ these growth factors could explain in part the cytomorphology of GCTB giant cells and the osteolytic behaviour of this tumour.

\section{ACKNOWLEDGEMENTS}

We would like to thank Chris Lowe for typing the manuscript. NAA conceived the study and analysed data. RT, TGK and HK carried out experiments and analysed data. All authors were involved in writing the paper and had final approval of the submitted and published versions. This study was supported by the Oxford NIHR, BRU and was undertaken as part of EuroBoNet, an EU-funded Network of Excellence.

\section{DISCLOSURE/CONFLICT OF INTEREST}

The authors declare no conflict of interest.

1. Fujikawa Y, Quinn JM, Sabokbar A, et al. The human osteoclast precursor circulates in the monocyte fraction. Endocrinology 1996; 137:4058-4060.

2. Quinn JM, Sabokbar A, Athanasou NA. Cells of the mononuclear phagocyte series differentiate into osteoclastic lacunar bone resorbing cells. J Pathol 1996;179:106-111.

3. Chambers TJ, Fuller K, McSheehy PM, et al. The effects of calcium regulating hormones on bone resorption by isolated human osteoclastoma cells. J Pathol 1985;145:297-305.

4. Lau YS, Sabokbar A, Gibbons CL, et al. Phenotypic and molecular studies of giant-cell tumors of bone and soft tissue. Hum Pathol 2005;36:945-954.

5. Quinn JM, Elliott J, Gillespie MT, et al. A combination of osteoclast differentiation factor and macrophage-colony stimulating factor is sufficient for both human and mouse osteoclast formation in vitro. Endocrinology 1998;139:4424-4427.

6. Felix R, Cecchini MG, Hofstetter W, et al. Impairment of macrophage colony-stimulating factor production and lack of resident bone marrow macrophages in the osteopetrotic op/op mouse. J Bone Miner Res 1990;5:781-789.

7. Yoshida $\mathrm{H}$, Hayashi $\mathrm{S}$, Kunisada $\mathrm{T}$, et al. The murine mutation osteopetrosis is in the coding region of the macrophage colony stimulating factor gene. Nature 1990;345:442-444.

8. Fuller K, Owens JM, Jagger CJ, et al. Macrophage colony-stimulating factor stimulates survival and chemotactic behavior in isolated osteoclasts. J Exp Med 1993;178:1733-1744.

9. Hodge JM, Kirkland MA, Nicholson GC. Multiple roles of M-CSF in human osteoclastogenesis. J Cell Biochem 2007;102:759-768.

10. Tsurukai T, Udagawa N, Matsuzaki K, et al. Roles of macrophagecolony stimulating factor and osteoclast differentiation factor in osteoclastogenesis. J Bone Miner Metab 2000;18:177-184.

11. Edwards M, Sarma U, Flanagan AM. Macrophage colony-stimulating factor increases bone resorption by osteoclasts disaggregated from human fetal long bones. Bone 1998;22:325-329.

12. Hattersley G, Dorey E, Horton MA, et al. Human macrophage colonystimulating factor inhibits bone resorption by osteoclasts disaggregated from rat bone. J Cell Physiol 1988;137:199-203.

13. Lees RL, Heersche JN. Macrophage colony stimulating factor increases bone resorption in dispersed osteoclast cultures by increasing osteoclast size. J Bone Miner Res 1999;14:937-945.

14. Begg SK, Radley JM, Pollard JW, et al. Delayed hematopoietic development in osteopetrotic (op/op) mice. J Exp Med 1993;177: 237-242.

15. Felix R, Hofstetter W, Wetterwald A, et al. Role of colony-stimulating factor-1 in bone metabolism. J Cell Biochem 1994;55:340-349.

16. Adamopoulos IE, Xia Z, Lau YS, et al. Hepatocyte growth factor can substitute for M-CSF to support osteoclastogenesis. Biochem Biophys Res Commun 2006;350:478-483.

17. Lean JM, Fuller K, Chambers TJ. FLT3 ligand can substitute for macrophage colony-stimulating factor in support of osteoclast differentiation and function. Blood 2001;98:2707-2713.

18. Niida S, Kaku M, Amano $\mathrm{H}$, et al. Vascular endothelial growth factor can substitute for macrophage colony-stimulating factor in the support of osteoclastic bone resorption. J Exp Med 1999;190:293-298.

19. Niida $\mathrm{S}$, Kondo T, Hiratsuka $\mathrm{S}$, et al. VEGF receptor 1 signaling is essential for osteoclast development and bone marrow formation in colony-stimulating factor 1-deficient mice. Proc Natl Acad Sci USA 2005;102:14016-14021.

20. Zheng $\mathrm{MH}$, Robbins $\mathrm{P}, \mathrm{Xu}$ J, et al. The histogenesis of giant cell tumour of bone: a model of interaction between neoplastic cells and osteoclasts. Histol Histopathol 2001;16:297-307. 
21. Aldridge SE, Lennard TW, Williams JR, et al. Vascular endothelial growth factor receptors in osteoclast differentiation and function. Biochem Biophys Res Commun 2005;335:793-798.

22. Hodge JM, Kirkland MA, Nicholson GC. GM-CSF cannot substitute for $\mathrm{M}-\mathrm{CSF}$ in human osteoclastogenesis. Biochem Biophys Res Commun 2004;321:7-12.

23. Lin $\mathrm{H}$, Lee $\mathrm{E}$, Hestir $\mathrm{K}$, et al. Discovery of a cytokine and its receptor by functional screening of the extracellular proteome. Science 2008;320: 807-811.

24. Baud'huin M, Renault R, Charrier C, et al. Interleukin-34 is expressed by giant cell tumours of bone and plays a key role in RANKL-induced osteoclastogenesis. J Pathol 2010;221:77-86.

25. Maes C, Coenegrachts L, Stockmans I, et al. Placental growth factor mediates mesenchymal cell development, cartilage turnover, and bone remodeling during fracture repair. J Clin Invest 2006;116:1230-1242.

26. Coenegrachts $\mathrm{L}$, Maes $\mathrm{C}$, Torrekens $\mathrm{S}$, et al. Anti-placental growth factor reduces bone metastasis by blocking tumor cell engraftment and osteoclast differentiation. Cancer Res 2010;70:6537-6547.

27. Zhang Q, Guo R, Lu Y, et al. VEGF-C, a lymphatic growth factor, is a RANKL target gene in osteoclasts that enhances osteoclastic bone resorption through an autocrine mechanism. J Biol Chem 2008; 283:13491-13499.
28. Salerno M, Avnet S, Alberghini M, et al. Histogenetic characterization of giant cell tumor of bone. Clin Orthop Relat Res 2008;466: 2081-2091.

29. Atkins GJ, Haynes DR, Graves SE, et al. Expression of osteoclast differentiation signals by stromal elements of giant cell tumors. J Bone Miner Res 2000;15:640-649.

30. Knowles HJ, Athanasou NA. Hypoxia-inducible factor is expressed in giant cell tumour of bone and mediates paracrine effects of hypoxia on monocyte-osteoclast differentiation via induction of VEGF. J Pathol 2008;215:56-66.

31. Hodge JM, Collier FM, Pavlos NJ, et al. M-CSF potently augments RANKL-induced resorption activation in mature human osteoclasts. PLoS One 2011;6:e21462.

32. Faccio $R$, Takeshita $S$, Colaianni $G$, et al. M-CSF regulates the cytoskeleton via recruitment of a multimeric signaling complex to c-Fms Tyr-559/697/721. J Biol Chem 2007;282:18991-18999.

33. Nakagawa M, Kaneda T, Arakawa T, et al. Vascular endothelial growth factor (VEGF) directly enhances osteoclastic bone resorption and survival of mature osteoclasts. FEBS Lett 2000;473:161-164.

34. Fuller K, Owens J, Chambers TJ. The effect of hepatocyte growth factor on the behaviour of osteoclasts. Biochem Biophys Res Commun $1995 ; 212: 334-340$ 